\title{
Emotional Expression in Simple Line Drawings of a Robot's Face Leads to Higher Offers in the Ultimatum Game
}

\author{
Kazunori Terada * and Chikara Takeuchi \\ Informatics Course, Department of Electrical, Electronics and Computer Engineering, Faculty of Engineering, Gifu University, \\ Gifu, Japan
}

In the present study, we investigated whether expressing emotional states using a simple line drawing to represent a robot's face can serve to elicit altruistic behavior from humans. An experimental investigation was conducted in which human participants interacted with a humanoid robot whose facial expression was shown on an LCD monitor that was mounted as its head (Study 1). Participants were asked to play the ultimatum game, which is usually used to measure human altruistic behavior. All participants were assigned to be the proposer and were instructed to decide their offer within 1 min by controlling a slider bar. The corners of the robot's mouth, as indicated by the line drawing, simply moved upward, or downward depending on the position of the slider bar. The results suggest that the change in the facial expression depicted by a simple line drawing of a face significantly affected the participant's final offer in the ultimatum game. The offers were increased by $13 \%$ when subjects were shown contingent changes of facial expression. The results were compared with an experiment in a teleoperation setting in which participants interacted with another person through a computer display showing the same line drawings used in Study 1 (Study 2). The results showed that offers were $15 \%$ higher if participants were shown a contingent facial expression change. Together, Studies 1 and 2 indicate that emotional expression in simple line drawings of a robot's face elicits the same higher offer from humans as a human telepresence does.

*Correspondence:

Kazunori Terada terada@gifu-u.ac.jp

Specialty section:

This article was submitted to Human-Media Interaction, a section of the journal Frontiers in Psychology

Received: 10 January 2017 Accepted: 21 April 2017 Published: 22 May 2017

Citation:

Terada K and Takeuchi C (2017) Emotional Expression in Simple Line Drawings of a Robot's Face Leads to Higher Offers in the Ultimatum Game.

Front. Psychol. 8:724.

doi: 10.3389/fpsyg.2017.00724

\section{INTRODUCTION}

Recently, there has been increasing interest and progress in robotic emotional expressions. A wide variety of methods for achieving emotional expression have been proposed (Bethel and Murphy, 2008), including facial expressions (Bartneck, 2003; Breazeal, 2004; Kanoh et al., 2004; Itoh et al., 2006; Matsui et al., 2010), speech (Kim et al., 2009a,b), body movement (Shimokawa and Sawaragi, 2001; Bethel and Murphy, 2007), and colors (Sugano and Ogata, 1996; Kim et al., 2009a,b; Terada et al., 2012). Leaving aside discussion regarding a robot's ability to possess genuine emotions, implementing a display of emotion in robots could be useful not only by increasing their friendliness but also by helping them to influence people without explicit language (Breazeal, 2003, 2004).

There have been studies on the effect of robotic emotions on human behavior (Cassell and Thorisson, 1999; Bickmore and Picard, 2005; Leyzberg et al., 2011); these focused on the taskoriented effects of emotions. Leyzberg et al. (2011) showed that robots that express emotions 
elicited better human teaching. A long-term experiment conducted by Bickmore and Picard (2005) showed that an agent with relational behavior, including social-emotional responses, contributed to increasing participants' positive attitude about exercise. While these studies revealed that robots with emotions positively affect human behavior, the nature, and essential function of these emotions have not been discussed. In the present study, we focused on the social functional aspect of emotions and experimentally investigated the effect of emotional expression as depicted through a simple line drawing of a face on human economic behavior.

Emotions control the behavior of an agent. For example, fear increases heart rate and muscle tension and drives an agent to escape from a situation; consequently, fear helps in avoiding dangerous situations. Emotions affect not only one's own behavior but also that of others. An angry individual, for example, usually obtains concessions from a competitor in a conflict situation (van Kleef et al., 2004; Sinaceur and Tiedens, 2006; van Kleef and Côté, 2007; van Dijk et al., 2008; van Kleef et al., 2008; Sell et al., 2009; Fabiansson and Denson, 2012; Reed et al., 2014). Positive emotions are considered to have evolved to maintain cooperative relationships (Trivers, 1971; Alexander, 1987; Frank, 1988; Scharlemann et al., 2001; Brown and Moore, 2002; Brown et al., 2003; Mehu et al., 2007; Reed et al., 2012; Mussel et al., 2013).

Altruism is a behavior that reduces the actor's wealth while increasing the wealth of the recipient, whereas cooperation is a process in which agents work together to gain common or mutual wealth. However, altruism can be considered to be asynchronous cooperative behavior by considering direct or indirect reciprocity (Nowak and Sigmund, 2005). In order to produce altruistic behavior, one must ignore the loss of one's own wealth. Positive emotions such as happiness and kindness that are elicited from another's facial expressions presumably compensate for the loss. Therefore, emotion is more important for long-term or indirect reciprocal relationships than short-term (one-shot) cooperative tasks. We used altruistic behavior as a measure of the function of the robot's facial expression because our focus is on the long-term human-robot relationship.

Researchers have been investigating whether people have a tendency to cooperate with robots (Nishio et al., 2012; Torta et al., 2013; Sandoval et al., 2016). Decision making in economic games such as the prisoners' dilemma and the ultimatum game is used to measure the cooperative attitude of participants. Nishio et al. (2012) have studied how the appearance of agents (computer, humanoid, android, or human) affects participants' cooperativeness. They conclude that although the appearance of agents does not affect cooperativeness, conversation with a human-like agent (android) leads people to be more cooperative. Torta et al. (2013) reported that rejection scores in the ultimatum game are higher in the case of a computer opponent than in the case of a human or robotic opponent, indicating that people might treat a robot as a reciprocal partner. Sandoval et al. (2016) showed that participants who interacted with a robot showed significantly less cooperation than when they interacted with a human in the prisoner's dilemma. Further, participants offered significantly less money in the ultimatum game to the robot than to the human agent, indicating that people tend to cooperate more with a human agent than with a robot.

From the above discussion, the following prediction could be derived: if robots offer emotional expression, people behave more cooperatively toward them. There are a few studies that examine the effect of the emotional expression of robots on human cooperative behavior in terms of economic behavior (de Melo et al., 2010, 2011). de Melo et al. (2010) conducted an experiment in which participants play the iterated prisoner's dilemma against two different virtual agents that play the tit-for-tat strategy but communicate different goal orientations (cooperative vs. individualistic) through their patterns of facial displays. They showed that participants were sensitive to differences in the facial displays and cooperated significantly more with the cooperative agent. de Melo et al. (2011), in another study, reported that participants conceded more to a virtual agent that expresses anger than to one that expresses happiness in a negotiation task.

The studies of de Melo et al. $(2010,2011)$ used human-like virtual character agents. In our study, we used a real robot with a simple line drawing of a face to remove realistic and biological human features from the agent's face (Terada et al., 2013). Most of the robots that are used in human-robot interaction studies have sophisticated facial expression mechanisms (Breazeal, 2004; Itoh et al., 2006; Matsui et al., 2010; Becker-Asano and Ishiguro, 2011; Mazzei et al., 2011). The underlying assumption is that mimicking real human facial expressions induces humans to emotionally respond as they would when interacting with a real human. However, studies have revealed that line drawing facial expressions are recognized to the same extent as a realistic face (Katsikitis, 1997; Britton et al., 2008), affect human altruistic behavior even they are slightly different (Brown and Moore, 2002), and are processed in the human brain in the same way as a human face (Britton et al., 2008) .

In the present study, we investigated whether a simple line drawing of a face is useful in human-robot interaction in terms of human-robot cooperative relationships. Terada et al. (2013) have showed that emotional expression by robots led people to behave more altruistically toward the robots even though the emotion was represented by simple line drawings. However, it is unclear whether this effect is the same extent as that of human-human interaction. In the present paper, we first show the results of human-robot condition reported in Terada et al. (2013) as Study 1. We then show the results of human-human condition (Study 2) and compare the results of these two studies.

The ultimatum game has been used to measure human altruistic behavior (Güth and Tietz, 1990; Sanfey et al., 2003; Oosterbeek et al., 2004; Xiao and Houser, 2005; van Dijk et al., 2008; Yamagishi et al., 2009). It is played by two players, a proposer and a responder, who are given the opportunity to split an allotment of money. The proposer has the right to divide the money and offer an amount to the responder. If the responder accepts the proposal, both players keep the money. If the responder rejects the proposal, neither player receives the money. The findings of a meta-analysis of 37 papers with 75 results from ultimatum game experiments showed that on average, the proposer offers $40 \%$ of the money to the responder, and $16 \%$ of the offers are rejected (Oosterbeek et al., 2004). 
In our study, all participants were assigned to be the proposer and were instructed to decide their offer within 1 min by controlling a slider bar. In the decision period, a change in the responder's facial expression was shown to the proposer (only in the change of facial expression condition), which is not a normal procedure in the ultimatum game. The communication before the decision is treated as cheap talk, which is costless and unverifiable preplay statements about private information and non-credible threats about future actions (Croson et al., 2003). Croson et al. (2003) showed that threats of future actions influenced bargaining outcomes.

The goal of the present study was to explore whether communication using the facial expression of robots is effective in establishing human-robot cooperative relationships. We used the offer in the ultimatum game as the measurement of cooperative attitude of human toward a robot. As a result, the effectiveness of facial expression of robots in human-robot cooperative relationship could be evaluated in terms of economic value.

Studies 1 and 2 were both conducted in accordance with the recommendations of the Ethical Guidelines for Medical and Health Research Involving Human Subjects provided by the Ministry of Education, Culture, Sports, Science, and Technology and the Ministry of Health, Labor, and Welfare in Japan with written informed consent from all subjects. All subjects gave written informed consent in accordance with the Declaration of Helsinki. The protocol was approved by the Medical Review Board of Gifu University Graduate School of Medicine.

\section{STUDY 1}

\subsection{Method}

\subsubsection{Participants}

Twenty-six healthy graduate and undergraduate students (15 male, 11 female, $M_{\text {age }}=19.62$ years, $S D_{\text {age }}=3.85$ years, age range: $18-24$ years) participated in the experiment. Participants were recruited through advertising on posters and via e-mail at the university. They were informed that they would be paid with a JPY 500 (approximately USD 5) book coupon for their time. All were ignorant of the purpose of the experiment.

\subsubsection{Experimental Design}

A single-factor two-level between-participants experimental design was used. Participants were randomly assigned to either a "change of facial expression" or a "static face" condition. All participants assumed the role of the proposer and were asked to determine their offer within $1 \mathrm{~min}$ by controlling the slider bar. The only difference between the two conditions was whether the corners of the mouth of the line drawing shown on an LCD monitor mounted on the robot moved upward or downward according to the position of the slider bar. In the initial state, a straight line segment represented the line drawing mouth.

\subsubsection{Apparatus}

The ultimatum game was played once. The proposer was given 100 points, which corresponds to JPY 1000 (approximately USD 10 ), as the amount to divide. The proposer was given $1 \mathrm{~min}$ to determine the offer (decision phase). During the decision phase, the proposer adjusted the offer by controlling the slider bar. Participants were informed that the game would be played against a humanoid robot that might react to the participant's offer through an LCD monitor mounted on the robot.

A GUI was used to determine the offer and to communicate the emotional state of the responder (see Figure 1). The proposer was asked to decide the offer within $1 \mathrm{~min}$ by moving a slider bar on the GUI, which was controlled by a gamepad connected to the computer.

\section{Static Face Condition}

The line drawing face did not change during the proposal period.

\section{Change of Facial Expression Condition}

The corners of the mouth of the line drawing moved upward and downward according to the position of and one second after the movement of the slider bar. This delay was inserted to prevent the participants from assuming that the responder was merely a simple computer program; an immediate mouth movement completely contingent on the proposer's action might strongly indicate artificiality. The software's calculation rate was $60 \mathrm{fps}$, the same as the monitor used to display the GUI.

Figure 2 shows the control points of a Bézier curve, which represented the line drawing of the mouth. The points P3 and $\mathrm{P} 4$ are the static points. The Y-coordinates of the points P0, P1, and P2 changed according to the position of the slider bar. Figure 3 illustrates examples of the facial expressions shown to the proposer as a function of the proposer's offer $x \in[0,100]$. If the slider bar moved to the right, the offer decreased and negative facial expressions, such as those shown in Figures 3A,B, were displayed.

Figure 4 shows the experimental system. We mounted an LCD monitor on a Robovie-X, a commercially available robot. Line drawings of facial expressions were shown on the mounted LCD monitor, which was connected to a laptop computer via a

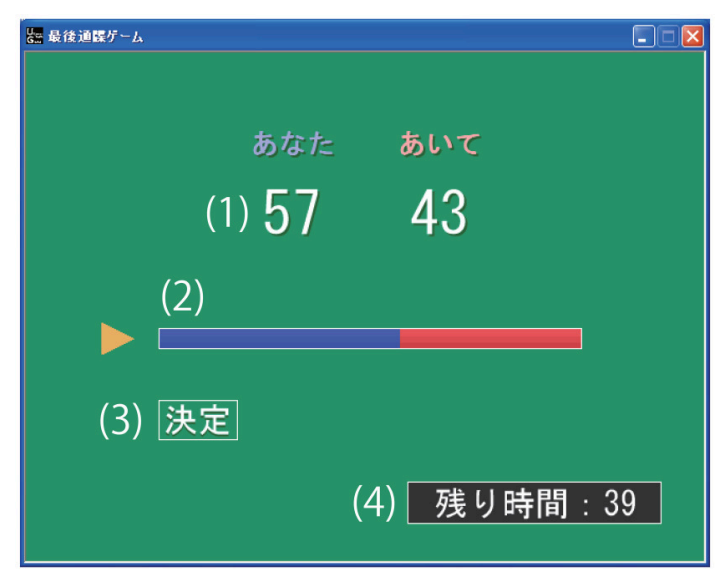

FIGURE 1 | Graphical user interface used by the proposer to determine the offer: (1) numerical representation of the offer, (2) slider bar to change the offer, (3) button for final decision, and (4) time remaining. 
USB cable. The laptop computer was also used to display the GUI, and a gamepad for controlling the slider bar on the GUI was connected to the laptop.

\subsubsection{Procedure}

In the experiment room, participants were asked to read an instruction sheet that stated the rules of the ultimatum game, how to use the interface, and that "the response of the responder will be shown on the head display." In addition, they were informed that they were assigned to be the proposer and that they would win additional money according to their score in the game.

After the proposal, the participants were not immediately informed of the responder's acceptance/rejection: they were first asked to complete a questionnaire to avoid the questionnaire responses being affected by the responder's decision. After completing the questionnaire, the participants were informed that they had all played as proposers against a computer program, and they were paid with an additional JPY 500 (approximately USD 5) book coupon, the amount of money that would be given if a 50:50 offer was accepted.

\subsubsection{Measurement and Analysis}

The offer was recorded every $0.5 \mathrm{~s}$. After the game, participants were asked to answer four 7-point Likert scale questions $(0=$ "definitely no" to 7 = "definitely yes"):

- Q1. Did you perceive emotions in the picture shown on the head of the robot?

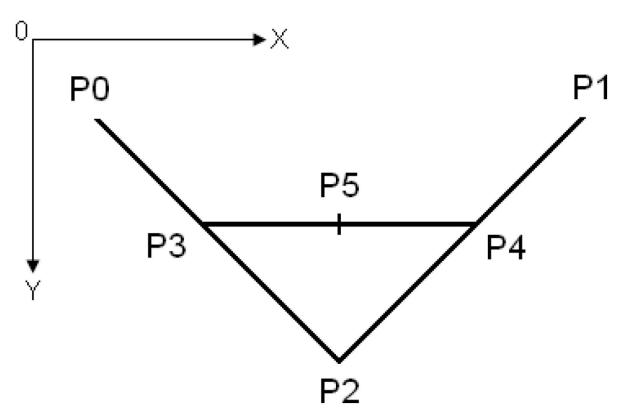

FIGURE 2 | Control points of the Bézier curve used to represent the mouth.
- Q2. Did you consider the responder's emotions when deciding your offer?

After answering the post-questionnaires, participants were asked whether they realized that they had been playing against a computer program.

The one-way analysis of variance (ANOVA) was used if the data were normally and homogeneously distributed. The Welch's ANOVA was used if the data were normally distributed, but the assumption of homogeneity of variance was violated. The Mann-Whitney $U$-test was used if the data were homogeneously distributed, but the assumption of normality was rejected. The Brunner-Munzel test was used if both the assumption of normality and the homogeneity of variance were violated.

\subsection{Results}

The mean durations for deciding an offer were $28.31 \mathrm{~s}$ ( $S D=$ $17.27)$ and $18.69 \mathrm{~s}(S D=14.26)$ in the change of facial expression and static face conditions, respectively. The one-way ANOVA, $F_{(1,24)}=2.40, p=0.13$, indicated that the difference was not statistically significant.

Figure 5 presents the mean final offers over participants in both conditions. Welch's ANOVA, $F_{(1,15.71)}=6.22, p<0.05$, showed that offers were higher in the change of facial expression condition $(M=51.62, S D=6.91)$ than in the static face condition $(M=38.69, S D=17.35)$.

Figure 6 displays the results of the post-experiment questionnaire. The Mann-Whitney $U$-tests, $U=18.5$, $z=3.46, p<0.001$, revealed that ratings for perceiving emotions from the line drawing were significantly higher in the change of facial expression condition than in the static face condition. The one-way ANOVA, $F_{(1,24)}=30.03, p<0.001$, revealed that ratings for the consideration of emotions were significantly higher in the change of facial expression condition than in the static face condition.

Ten out of 13 participants in the change of facial expression condition realized that they had played against a computer program that generates a simple mouth movement completely contingent on the participants' action.

\subsection{Discussion}

The results show that offers were higher in the change of facial expression condition than in the static face condition, confirming that emotional expression by robots led participants to behave more altruistically toward the robots even though the emotion
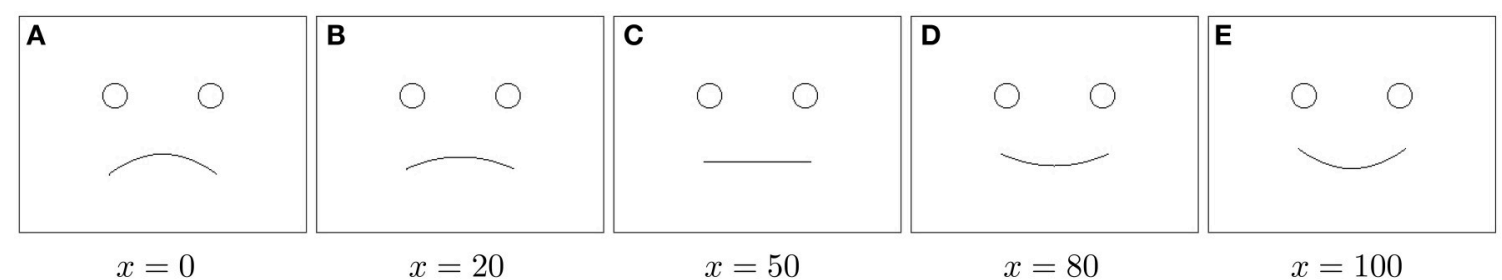

FIGURE 3 | Examples of the facial expressions displayed to the proposer as a function of the proposer's offer $x \in[\mathbf{0}, 100]$. (A) $x=0$. (B) $x=20$. (C) $x=$ 50. (D) $x=80$. (E) $x=100$. 


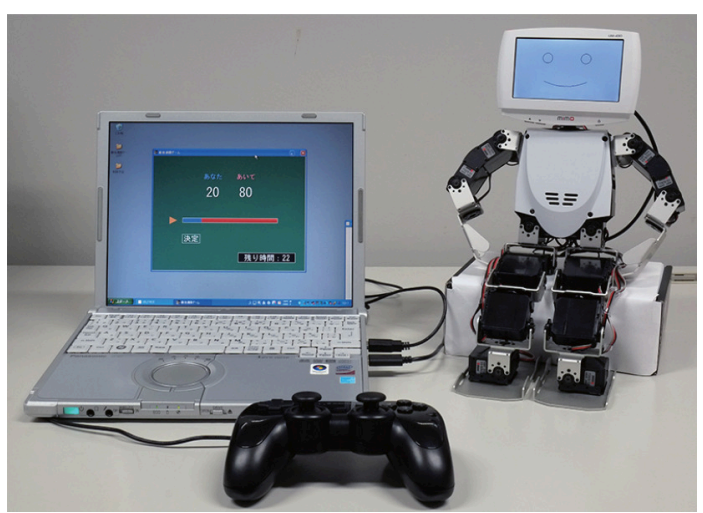

FIGURE 4 | System used in our experiment.

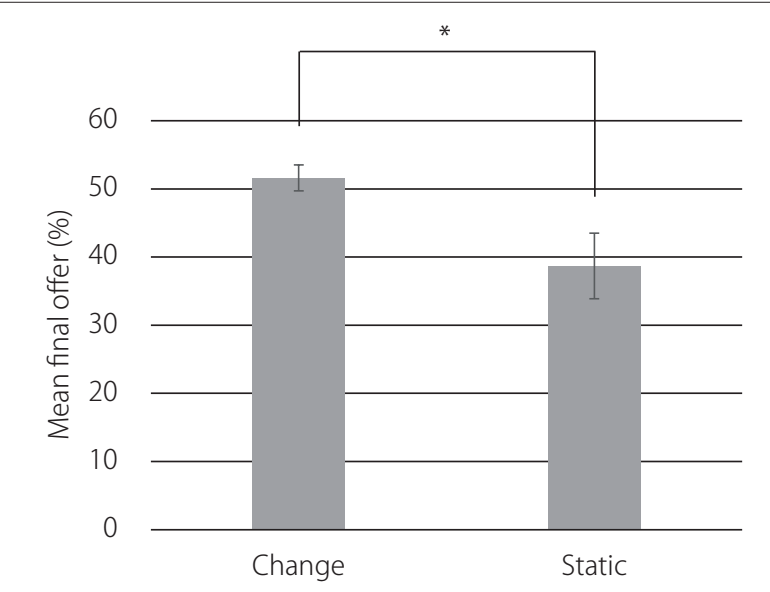

FIGURE 5 | Mean final offers over participants in both conditions. Error bars indicate standard errors. ${ }^{*} p<0.05$.

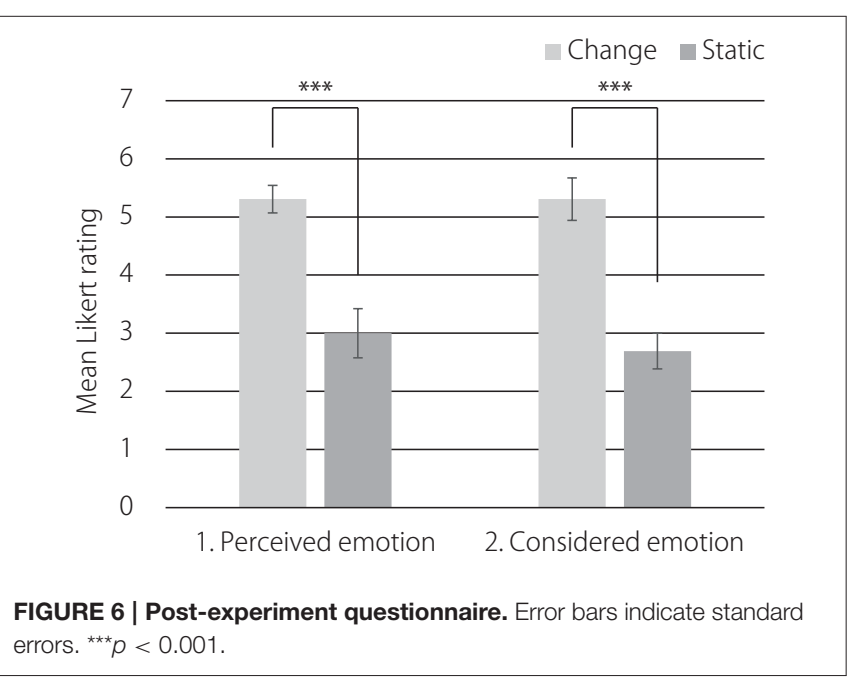

was represented by simple line drawings. The results of the post-experiment questionnaires support the behavioral result that the $12.92 \%$ gap between the two conditions was caused by the emotions that participants recognized from the change of facial expressions exhibited by the line drawing. Participants in the change of facial expression condition gave higher ratings, an average of 5.30, to the question "Did you perceive emotions in the picture located on the upper right of the GUI?" than did participants in the static face condition. There was a large gap, an average of 2.30, in the Q1 rating between the two conditions, which indicates that perceiving emotions caused the participants' altruistic behavior.

The conditions differed only in whether the corners of the mouth of the line drawing in the GUI changed. However, we did not explicitly inform participants that the line drawing symbolized a face or that the position of the bar represented the position of the corners of the mouth. The participants arbitrarily attributed a facial property to the geometric line drawings and attributed emotions to variable Bézier curves. According to Ekman (2003), a convex mouth shape, in which the corners of the lips curl downward, indicates sadness, and a concave mouth shape, in which corners of the lips move upward, indicates happiness. Although, we did not identify the emotions that participants perceived from the line drawings, the universality of facial expressions supports the assumption that participants recognized sadness when they were shown a convex mouth and happiness when they were shown a concave mouth.

Our results show that although a substantial number of participants $(78 \%)$ in the change of facial expression condition realized that the mouth movement was controlled by a computer program, the effect of facial expression was still observed. de Melo et al. (2011) reported similar findings from a study in which participants were involved in a negotiation with computer agents. Taken together, these findings imply that facial expressions are effective in inducing people to cooperate with robots even though they know that the expressions are controlled by a program.

A meta-analysis of 75 results from ultimatum game experiments revealed that the proposer usually offers $40 \%$ of the money to the responder (Oosterbeek et al., 2004). However, participants in the change of facial expression condition offered an average of $51.62 \%$ of the money. This indicates that the offer increased by approximately $10 \%$ when people were shown changes of facial expression corresponding to their offer. By contrast, participants in the static face condition offered an average of $38.69 \%$. This value roughly corresponds to that offered in the earlier studies that included no emotional interaction in their experimental setting.

There are two potential reasons why participants in the change of facial expression condition offered approximately 50:50, which is a fair offer. The first is the impression that the responder has the capability to respond emotionally, which is formed by the dynamic change of facial expression in response to the participant's operation. In this case, the facial expression itself does not have an absolute meaning: simply perceiving adaptivity or the ability to respond to the user's input might be lead to a fair offer. The second reason is a neutral face. In our experimental setting, a neutral face, in which the mouth was represented by a straight line, was displayed to participants when the offer was 50\%. Participants 
could adjust the slider bar to make the facial expression neutral. Further investigation, in which a neutral face does not correspond to a $50 \%$ offer, is needed to test these two hypotheses.

Our results do not identify whether positive or negative emotion contributed to an increase in the offer. It is known that expressing anger can elicit concessions from others (van Kleef et al., 2004; Sinaceur and Tiedens, 2006; van Kleef and Côté, 2007; van Dijk et al., 2008; van Kleef et al., 2008; Sell et al., 2009; Fabiansson and Denson, 2012; Reed et al., 2014), while happiness can elicit altruism (Scharlemann et al., 2001; Brown and Moore, 2002; Brown et al., 2003; Mehu et al., 2007; Mussel et al., 2013). These findings suggest that both the negative and positive expressions shown in our experiment might have contributed to the proposer raising the offer.

Croson et al. (2003) showed that threats of future actions influenced bargaining outcomes. The negative emotional expression that was contingently presented when a low offer was proposed might have played the role of cheap talk.

\section{STUDY 2}

Study 2 was conducted to compare the result of Study 1 with those from a study in which participants played the game against a human responder in a teleoperation setting through a computer display. The aim of this study was to determine whether the altruistic behavior induced by the robot's facial expression is also induced by a facial expression controlled by a human.

\subsection{Method}

\subsubsection{Participants and Experimental Design}

Forty healthy graduate and undergraduate students (35 male, 5 female, $M_{\text {age }}=21.38$ years, $S D_{\text {age }}=1.51$ years, age range: $18-$ 23 years) participated in the experiment. All participants were ignorant of the purpose of the experiment.

As in Study 1, a single-factor two-level between-participants experimental design was used. Participants were randomly assigned to either a "static face" or a "change of facial expression" condition.

\subsubsection{Apparatus}

The apparatus used was identical to that used in Study 1 except that the facial expression was shown on the upper right area of the GUI as shown in Figure 7.

\subsubsection{Procedure}

The procedure was identical to that used in Study 1, except for the following changes. The experiment was conducted on two participants who knew each other. The two participants came to the experiment together and were taken to different rooms. In their different rooms, they were asked to read the instruction paper, and both participantswere informed that they were assigned to be the proposer. Thus, all participants played the role of the proposer without knowing it. They were informed that "the response of your partner will be shown on the upper right

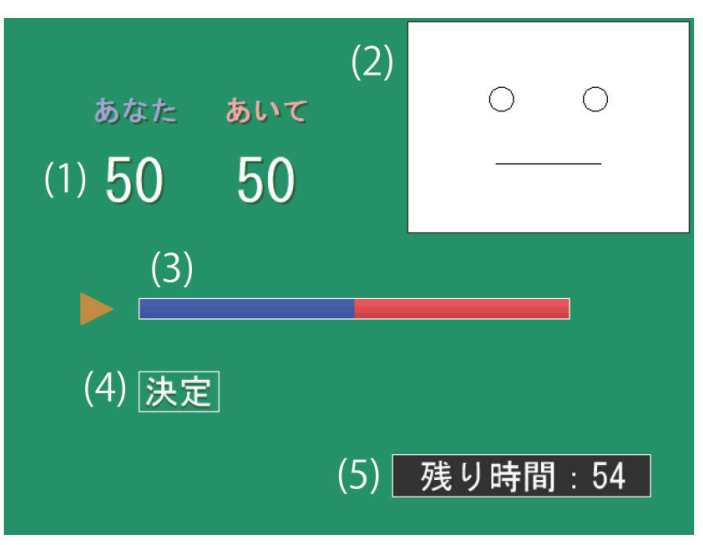

FIGURE 7 | Graphical user interface used in Study 2.

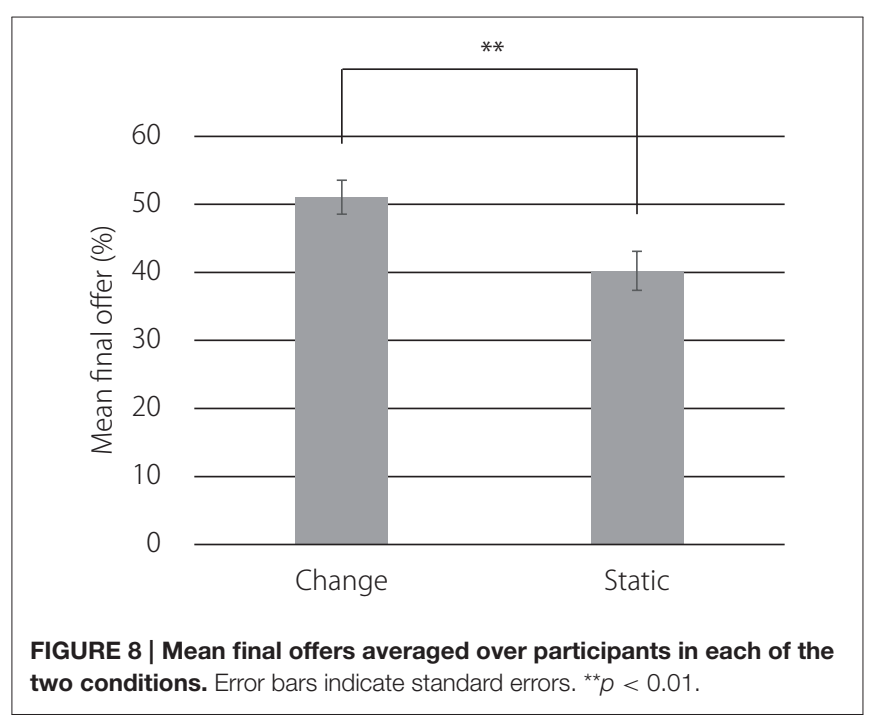

area of the interface." The facial expression was automatically changed based on the position of the slider bar controlled by the participant, as in Study 1.

\subsection{Results}

The data of one participant in each of the two conditions were excluded because they reported that they realized that they were playing against a computer program.

The mean durations spent deciding the amount of the offer were $50.31 \mathrm{~s}(S D=12.55)$ and $46.47 \mathrm{~s}(S D=14.68)$ in the facial expression change condition and static face condition, respectively. The Mann-Whitney $U$-tests, $U=162, z=0.54$ $p=0.59$, show that no statistically significant difference was observed.

Figure 8 presents the mean final offers averaged over participants in each of the two conditions. Error bars indicate standard errors of the mean value. The Mann-Whitney $U$-tests, $U=92, z=2.61, p<0.01$, show that offers were higher in the facial expression change condition $(M=51.05, S D=10.88)$ than in the static face condition $(M=40.21, S D=12.48)$. 


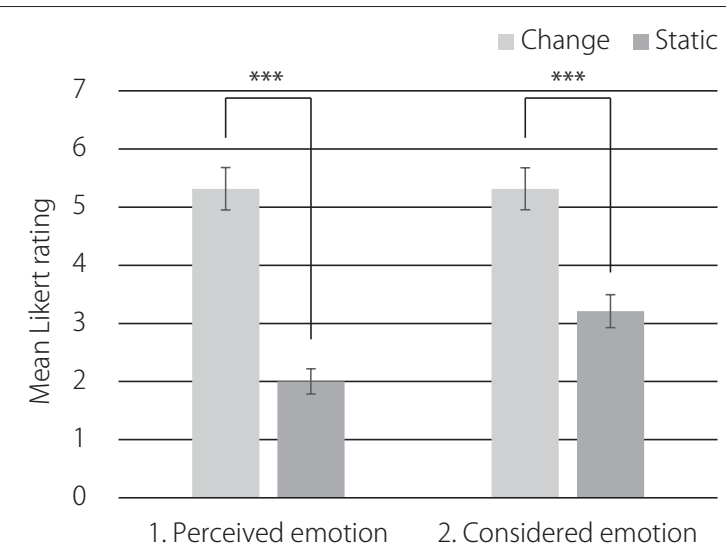

FIGURE 9 | Post-experiment questionnaire. Error bars indicate standard errors. ${ }^{\star * *} p<0.001$.

Figure 9 shows the results of the post-experiment questionnaire. The Brunner-Munzel test, $W=9.7, p<0.01$, revealed that the ratings for perceiving emotions from the linedrawing of a face were significantly higher in the facial expression condition than in the static face condition. The Mann-Whitney $U$-tests, $U=47.5, z=3.95, p<0.001$, revealed that ratings for ratings for considering this emotion were significantly higher in the facial expression condition than in the static face condition.

\subsection{Discussion}

The results show that offers were higher in the change of facial expression than in the static face condition, confirming that emotional expression given by an online responder through an avatar face composed of simple lines led participants to behave more altruistically to the responder. The results of the postexperiment questionnaires support the behavioral result.

\section{GENERAL DISCUSSION}

The behavioral and questionnaire results for Study 2 were similar to those of Study 1, confirming that emotional expression conveyed through simple line drawings representing a robot's face has the function of eliciting altruistic behavior from humans to the same extent as human telepresence.

However, it appears that the duration of time spent deciding the offer amount for a human responder was longer than that for a robot. This indicates that those participants who played the game against a human took more time to find the point of compromise. Despite this fact, interestingly, the mean final offers were almost the same between Study 1 and Study 2. It is possible that humans have a cognitive tendency to treat robots as non-negotiable partners and that this leads to a shorter duration of time spent exploring the point of compromise. However, the facial expression of the robot might have suppressed this cognitive tendency and led the participants to be more altruistic.

The results of our studies are consistent with those of previous studies (de Melo et al., 2010, 2011). The studies of de Melo et al. $(2010,2011)$ and ours all showed that the emotional expressions of artificial agents are effective in inducing humans to cooperate. de Melo et al. $(2010,2011)$ used a human-like virtual agent, whereas we used a real robot with a simple line drawing depicting its face. This implies that sophisticated human-likeness is not necessarily needed for a cooperative relationship to develop between robots and humans. This might be because facial expressions, even the face is a line drawing, are processed subcortically (Johnson, 2005; Britton et al., 2008). Nishio et al. (2012) have conducted experiments with an android robot that has a highly human-like appearance and concluded that the appearance of the agent does not affect cooperativeness. From these results, we would suggest that the ability to interact is more important than a human-like appearance for an artificial agent to develop a cooperative relationship with a human.

A substantial number of studies have shown that in economic games played by humans, facial expressions affect the decision to cooperate or not regardless of the type of game [e.g., ultimatum games (Mussel et al., 2014), prisoner's dilemma (Reed et al., 2012), dictator games (Brown and Moore, 2002), and trust games (Tortosa et al., 2013)]. Furthermore, whereas de Melo et al. (2010) used the prisoner's dilemma, we used an ultimatum game, and both studies show that the emotional expressions of artificial agents are effective in inducing humans to cooperate. Overall, it is possible that the emotional expressions of artificial agents are useful for building cooperative relationships with humans regardless of the type of game. However, long-term field study should be conducted to investigate whether the emotional expression contributes to the initiation and maintenance of real human-robot cooperative relationships.

Some limitations occur in the present study. First, while our participants were selected from a small, culturally homogeneous population and the gender ratio was not controlled, studies have suggested that culture (Russell, 1994; Hess et al., 2000; Mandal and Ambady, 2004) and gender (Hess et al., 2000; Mussel et al., 2014) influence the expression and interpretation of emotions. Larger and more diverse samples should be used to examine gender and cultural effects on human-robot cooperative relationships mediated by emotional expressions. Second, we used a small humanoid robot, and its facial expression was shown on an LCD monitor that was mounted as its head. Further, investigation using various types of robots such as life-sized humanoid robots and robots with sophisticated facial expression mechanisms should be performed to generalize the findings.

\section{AUTHOR CONTRIBUTIONS}

KT designed the experiments; CT performed the experiments; $\mathrm{KT}$ and $\mathrm{CT}$ analyzed the data; and KT wrote the manuscript.

\section{ACKNOWLEDGMENTS}

This study was partially supported by JSPS KAKENHI No. 15H02735 and "Cognitive Interaction Design" (No.26118005). We would like to thank IEEE for permission to partially reprint from Terada et al. (2013). 


\section{REFERENCES}

Alexander, R. D. (1987). The Biology of Moral Systems. New York, NY: Aldine De Gruyter.

Bartneck, C. (2003). "Interacting with an embodied emotional character," in Proceedings of the 2003 International Conference on Designing Pleasurable Products and Interfaces, DPPI '03 (New York, NY: ACM), 55-60.

Becker-Asano, C., and Ishiguro, H. (2011). "Evaluating facial displays of emotion for the android robot geminoid f," in IEEE Workshop on Affective Computational Intelligence (WACI) (Paris), 1-8.

Bethel, C. L., and Murphy, R. R. (2007). "Non-facial/non-verbal methods of affective expression as applied to robot-assisted victim assessment," in Proceedings of the 2nd ACM/IEEE International Conference on Human-Robot Interaction, HRI '07 (New York, NY: ACM), 287-294.

Bethel, C. L., and Murphy, R. R. (2008). Survey of non-facial/non-verbal affective expressions for appearance-constrained robots. IEEE Trans. Syst. Man Cybernet. C Appl. Rev. 38, 83-92. doi: 10.1109/TSMCC.2007. 905845

Bickmore, T. W., and Picard, R. W. (2005). Establishing and maintaining longterm human-computer relationships. ACM Trans. Comp. Hum. Interact. 12, 293-327. doi: 10.1145/1067860.1067867

Breazeal, C. (2003). Emotion and sociable humanoid robots. Int. J. Hum. Comp. Stud. 59, 119-155. doi: 10.1016/S1071-5819(03)00018-1

Breazeal, C. (2004). Function meets style: insights from emotion theory applied to HRI. IEEE Trans. Syst. Man. Cybernet. C Appl. Rev. 34, 187-194. doi: 10.1109/TSMCC.2004.826270

Britton, J. C., Shin, L. M., Barrett, L. F., Rauch, S. L., and Wright, C. I. (2008). Amygdala and fusiform gyrus temporal dynamics: responses to negative facial expressions. BMC Neurosci. 9:44. doi: 10.1186/1471-2202-9-44

Brown, W. M., and Moore, C. (2002). Chapter 3: smile asymmetries and reputation as reliable indicators of likelihood to cooperate: an evolutionary analysis," in Advances in Psychology Research, Vol. 11 (New York, NY: Nova Science Publishers), 59-78.

Brown, W. M., Palameta, B., and Moore, C. (2003). Are there nonverbal cues to commitment? an exploratory study using the zeroacquaintance video presentation paradigm. Evol. Psychol. 1, 42-69. doi: $10.1177 / 147470490300100104$

Cassell, J., and Thorisson, K. R. (1999). The power of a nod and a glance: envelope vs. emotional feedback in animated conversational agents. Appl. Artif. Intell. 13, 519-538.

Croson, R., Boles, T., and Murnighan, J. K. (2003). Cheap talk in bargaining experiments: lying and threats in ultimatum games. J. Econ. Behav. Organ. 51, 143-159. doi: 10.1016/S0167-2681(02)00092-6

de Melo, C. M., Carnevale, P., and Gratch, J. (2010). “The influence of emotions in embodied agents on human decision-making," in Intelligent Virtual Agents, 10th International Conference, IVA 2010, Philadelphia, PA, USA. Proceedings, eds J. Allbeck, N. Badler, T. Bickmore, C. Pelachaud, and A. Safonova (Berlin; Heidelberg: Springer), 357-370. Available online at: http://www.springer.com/ us/book/9783642158919\# otherversion $=9783642158926$

de Melo, C. M., Carnevale, P., and Gratch, J. (2011). "The effect of expression of anger and happiness in computer agents on negotiations with humans," in The 10th International Conference on Autonomous Agents and Multiagent Systems, Vol. 3, AAMAS '11, ed S. C. Richland (Taipei: International Foundation for Autonomous Agents and Multiagent Systems), 937-944.

Ekman, P. (2003). Emotions Revealed: Recognizing Faces and Feelings to Improve Communication and Emotional Life. New York, NY: Times Books.

Fabiansson, E. C., and Denson, T. F. (2012). The effects of intrapersonal anger and its regulation in economic bargaining. PLoS ONE 7:e51595. doi: 10.1371/journal.pone.0051595

Frank, R. H. (1988). Passions within Reason: The Strategic Role of the Emotions. New York, NY: W.W. Norton \& Co.

Güth, W., and Tietz, R. (1990). Ultimatum bargaining behavior : a survey and comparison of experimental results. J. Econ. Psychol. 11, 417-449.

Hess, U., Blairy, S., and Kleck, R. E. (2000). The influence of facial emotion displays, gender, and ethnicity on judgments of dominance and affiliation. J. Nonverb. Behav. 24, 265-283. doi: 10.1023/A:1006623213355

Itoh, K., Miwa, H., Nukariya, Y., Zecca, M., Takanobu, H., Roccella, S., et al. (2006). "Mechanisms and functions for a humanoid robot to express human-like emotions," in Proceedings of the 2006 IEEE International Conference on Robotics and Automation (Orlando, FL), 4390-4392.

Johnson, M. H. (2005). Subcortical face processing. Nat. Rev. Neurosci. 6, 766-774 doi: $10.1038 / \mathrm{nrn} 1766$

Kanoh, M., Kato, S., and Itoh, H. (2004). "Facial expressions using emotional space in sensitivity communication robot "ifbot", in IEEE/RSJ International Conference on Intelligent Robots and Systems, 2004 (IROS 2004), Vol. 2 (Sendai), 1586-1591.

Katsikitis, M. (1997). The classification of facial expressions of emotion: a multidimensional-scaling approach. Perception 26, 613-626.

Kim, E. H., Kwak, S., and Kwak, Y. K. (2009a). "Can robotic emotional expressions induce a human to empathize with a robot?" in The 18th IEEE International Symposium on Robot and Human Interactive Communication, 2009, RO-MAN 2009 (IEEE), 358-362.

Kim, E. H., Kwak, S. S., Han, J., and Kwak, Y. K. (2009b). "Evaluation of the expressions of robotic emotions of the emotional robot, "mung", in Proceedings of the 3rd International Conference on Ubiquitous Information Management and Communication, ICUIMC '09 (New York, NY: ACM), 362-365.

Leyzberg, D., Avrunin, E., Liu, J., and Scassellati, B. (2011). "Robots that express emotion elicit better human teaching," in HRI '11: Proceeding of the 6th ACM/IEEE International Conference on Human Robot Interaction, HRI '11 (New York, NY: ACM), 347-354.

Mandal, M. K., and Ambady, N. (2004). Laterality of facial expressions of emotion: Universal and culture-specific influences. Behav. Neurol. 15, 23-34. doi: 10.1155/2004/786529

Matsui, Y., Kanoh, M., Kato, S., Nakamura, T., and Itoh, H. (2010). A model for generating facial expressions using virtual emotion based on simple recurrent network. J. Adv. Comput. Intell. Intellig. Inform. 14, 453-463. doi: 10.20965/jaciii.2010.p0453

Mazzei, D., Lazzeri, N., Billeci, L., Igliozzi, R., Mancini, A., Ahluwalia, A., Muratori, F., and Rossi, D. D. (2011). "Development and evaluation of a social robot platform for therapy in autism," in Annual International Conference of the IEEE Engineering in Medicine and Biology Society, EMBC, 2011 (Boston, MA), 4515-4518.

Mehu, M., Grammer, K., and Dunbar, R. I. (2007). Smiles when sharing. Evol. Hum. Behav. 28, 415-422. doi: 10.1016/j.evolhumbehav.2007.05.010

Mussel, P., Göritz, A. S., and Hewig, J. (2013). The value of a smile: facial expression affects ultimatum-game responses. Judgm. Decis. Making 8, 381-385. Available online at: http://journal.sjdm.org/12/12817/jdm12817.html

Mussel, P., Hewig, J., Allen, J. J. B., Coles, M. G. H., and Miltner, W. (2014). Smiling faces, sometimes they don't tell the truth: facial expression in the ultimatum game impacts decision making and event-related potentials. Psychophysiology 51, 358-363. doi: 10.1111/psyp.12184

Nishio, S., Ogawa, K., Kanakogi, Y., Itakura, S., and Ishiguro, H. (2012). "Do robot appearance and speech affect people's attitude? Evaluation through the ultimatum game," in The 21th IEEE International Symposium on Robot and Human Interactive Communication (RO-MAN2012) (Paris), 809-814.

Nowak, M. A., and Sigmund, K. (2005). Evolution of indirect reciprocity. Nature 437, 1291-1298. doi: 10.1038/nature04131

Oosterbeek, H., Sloof, R., and van de Kuilen, G. (2004). Cultural differences in ultimatum game experiments: evidence from a meta-analysis. Exp. Econ. 7, 171-188. doi: 10.1023/B:EXEC.0000026978.14316.74

Reed, L. I., DeScioli, P., and Pinker, S. A. (2014). The commitment function of angry facial expressions. Psychol. Sci. 25, 1511-1517. doi: $10.1177 / 0956797614531027$

Reed, L. I., Zeglen, K. N., and Schmidt, K. L. (2012). Facial expressions as honest signals of cooperative intent in a one-shot anonymous prisoner's dilemma game. Evol. Hum. Behav. 33, 200-209. doi: 10.1016/j.evolhumbehav.2011.09.003

Russell, J. A. (1994). Is there universal recognition of emotion from facial expression? a review of the cross-cultural studies. Psychol. Bull. 115, 102-141.

Sandoval, E. B., Brandstetter, J., Obaid, M., and Bartneck, C. (2016). Reciprocity in human-robot interaction: a quantitative approach through the prisoner's dilemma and the ultimatum game. Int. J. Soc. Robot. 8, 303-317. doi: 10.1007/s12369-015-0323-x

Sanfey, A. G., Rilling, J. K., Aronson, J. A., Nystrom, L. E., and Cohen, J. D. (2003). The neural basis of economic decision-making in the ultimatum game. Science 300, 1755-1758. doi: 10.1126/science.1082976 
Scharlemann, J. P. W., Eckel, C. C., Kacelnik, A., and Wilson, R. K. (2001). The value of a smile: game theory with a human face. J. Econ. Psychol. 22, 617-640. doi: 10.1016/S0167-4870(01)00059-9

Sell, A., Tooby, J., and Cosmides, L. (2009). Formidability and the logic of human anger. Proc. Natl. Acad. Sci. U.S.A. 106, 15073-15078. doi: 10.1073 /pnas.0904312106

Shimokawa, T., and Sawaragi, T. (2001). "Acquiring communicative motor acts of social robot using interactive evolutionary computation," in IEEE International Conference on Systems, Man, and Cybernetics, Vol. 3 (Anchorage, AK), 1396-1401.

Sinaceur, M., and Tiedens, L. Z. (2006). Get mad and get more than even: when and why anger expression is effective in negotiations. J. Exp. Soc. Psychol. 42, 314-322. doi: 10.1016/j.jesp.2005.05.002

Sugano, S., and Ogata, T. (1996). Emergence of mind in robots for human interface - research methodology and robot model. IEEE Int. Conf. Robot. Automat. 2, 1191-1198.

Terada, K., Takeuchi, C., and Ito, A. (2013). "Effect of emotional expression in simple line drawings of a face on human economic behavior," in The 22th IEEE International Symposium on Robot and Human Interactive Communication (RO-MAN 2013) (Gyeongju), 51-56.

Terada, K., Yamauchi, A., and Ito, A. (2012). "Artificial emotion expression for a robot by dynamic color change," in The 21th IEEE International Symposium on Robot and Human Interactive Communication (RO-MAN 2012) (Paris), 314-321.

Torta, E., van Dijk, E., Ruijten, P. A. M., and Cuijpers, R. H. (2013). The Ultimatum Game as Measurement Tool for Anthropomorphism in HumanRobot Interaction. Cham: Springer International Publishing.

Tortosa, M. I., Strizhko, T., Capizzi, M., and Ruz, M. (2013). Interpersonal effects of emotion in a multi-round trust game. Psicológica 34, 179-198. Available online at: https://www.uv.es/psicologica/articulos2.13/3Tortosa.pdf
Trivers, R. L. (1971). The evolution of reciprocal altruism. Q. Rev. Biol. 46, 35-57. van Dijk, E., van Kleef, G. A., Steinel, W., and van Beest, I. (2008). A social functional approach to emotions in bargaining: when communicating anger pays and when it backfires. J. Pers. Soc. Psychol. 94, 600-614. doi: 10.1037/0022-3514.94.4.600

van Kleef, G. A., and Côté, S. (2007). Expressing anger in conflict: when it helps and when it hurts. J. Appl. Psychol. 92, 1557-1569. doi: 10.1037/0021-9010.92.6.1557

van Kleef, G. A., Dreu, C. K. W. D., and Manstead, A. S. R. (2004). The interpersonal effects of anger and happiness in negotiations. J. Pers. Soc. Psychol. 86, 57-76. doi: 10.1037/0022-3514.86.1.57

van Kleef, G. A., van Dijk, E., Steinel, W., Harinck, F., and van Beest, I. (2008). Anger in social conflict: cross-situational comparisons and suggestions for the future. Group Decis. Negot. 17, 13-30. doi: 10.1007/s10726-007-9092-8

Xiao, E., and Houser, D. (2005). Emotion expression in human punishment behavior. Proc. Natl. Acad. Sci. U.S.A. 102, 7398-7401. doi: 10.1073/pnas.0502399102

Yamagishi, T., Horita, Y., Takagishi, H., Shinada, M., Tanida, S., and Cook, K. S. (2009). The private rejection of unfair offers and emotional commitment. Proc. Natl. Acad. Sci. U.S.A. 106, 11520-11523. doi: 10.1073/pnas.0900636106

Conflict of Interest Statement: The authors declare that the research was conducted in the absence of any commercial or financial relationships that could be construed as a potential conflict of interest.

Copyright (c) 2017 Terada and Takeuchi. This is an open-access article distributed under the terms of the Creative Commons Attribution License (CC BY). The use, distribution or reproduction in other forums is permitted, provided the original author(s) or licensor are credited and that the original publication in this journal is cited, in accordance with accepted academic practice. No use, distribution or reproduction is permitted which does not comply with these terms. 